\title{
TO IMPROVE DAILY PRODUCTION OUPUT Using Lean Manufacturing Approach At PT. XYZ Batam
}

\author{
Dadang Redantan \\ Industrial Engineering Program, Faculty of Engineering, University of Riau Kepulauan (UNRIKA) \\ Jl. Letjend Suprapto, Bukit Tempayan, Batu Aji, Kota Batam, Kepulauan Riau 29425 \\ E-Mail : Dadang.redantan@yahoo.co.id
}

\begin{abstract}
ABSTRAK
Penelitian ini bertujuan untuk meningkatkan output produksi harian tipe LGA Sirkuit Terpadu (IC) di PT. XYZ Batam untuk memenuhi permintaan pelanggan. Metode yang digunakan adalah Lean Manufacturing. Hasil yang ditunjukkan dengan menghilangkan beberapa limbah akan meningkatkan kapasitas.
\end{abstract}

Kata kunci : Produksi Ouput, Pengiriman On-time, Balancing, Produksi IC, Inspeksi Akhir.

\section{INTRODUCTION}

PT. XYZ is an electronic manufacturing company who produce Integraged Circuit (IC). This company in the daily operation will produce the output using make to order strategy. As long as customer demand equal to or less than the existing production capacity, company should have no issue to serve customer demand.

One of the main customer suddently request output more than existing capacity, existing capacity is only set for 220,000 unit per day while customer demand is 300,000 unit per day.

This research mean to apply Lean Manufacturing concepts to find out all the possibility of waste in production process and eliminate wast to improve daily production output to meet customer demand.

\section{METHODOLOGY OF RESEARCH}

\subsection{Lean Manufacturing}

Lean Manufacturing is systematic approach to eliminate wast and change the process. This will reach by identify and eliminate waste with continuous improvement (AP, Wisnu and Partner, 2013) Lean Manufacturing motivate to make flexibility in production system which able to adopt in customer demand with sleam production and low inventory stock (Gaspersz, Vincent, 2007). Lean Manufacturing will use to eliminate waste and create balance production capacity to meet customer demand.

Lean Manufacturing has become common theme in today's manufacturing environment, for both commercial and defense industries. Lean Manufacturing is the common name for concepts exemplified by the Toyota Production System, with the overall philosophy of eliminating the source of waste in the manufacturing process (Altarum et all).

There are 8 type of waste which summary by word of DOWN TIME, consist of defects, over production, waiting, nonproduction hour, transportation, inventory in excess, motion and extra processing.

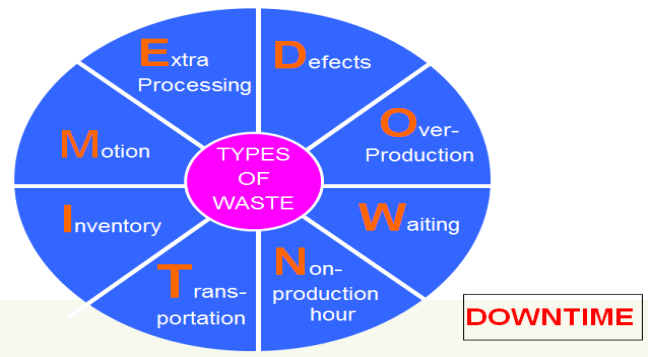

Figure 1. Eight (8) Type of Waste Which Summary by word of DOWN TIME. 
Table 1. Customer Demand July December 2015

\begin{tabular}{lcccccc}
\hline Pacakge & Jul-15 & Aug-15 & Sep-15 & Oct-15 & Nov-15 & Dec-15 \\
\hline LGA IC & 6.000 .000 & 6.000 .000 & 6.000 .000 & 6.000 .000 & 9.000 .000 & 9.000 .000 \\
\hline
\end{tabular}

Table 2. Delivery to Customer July $\sim$ December 2015

\begin{tabular}{lcccccc}
\hline Pacakge & Jul-15 & Aug-15 & Sep-15 & Oct-15 & Nov-15 & Dec-15 \\
\hline LGA IC & 5.950 .000 & 6.000 .000 & 5.950 .000 & 6.000 .000 & 6.600 .000 & 6.600 .000 \\
\hline
\end{tabular}

\section{RESULT AND DESCRIPTION OF RESULT}

Following is all the flow for IC LGA production from wafer incoming inspection and end by Final Visual Inspection and Packing the product (Figure 1).

Base on the data from Customer Service Department, below is customer demand data from July 2015 up to December 2015.
We can see there is an increase of demand in November 2015 from average of $6,000,000$ units per month become 9,000,000 units per month (Table 1).

Base on existing production capacity condition, company not able to meet all the customer requirement. Especially for November 2015 and December 2015, company only able to deliver $6,600,000$ unit vs actual demand 9,000,000 units (Table 2).

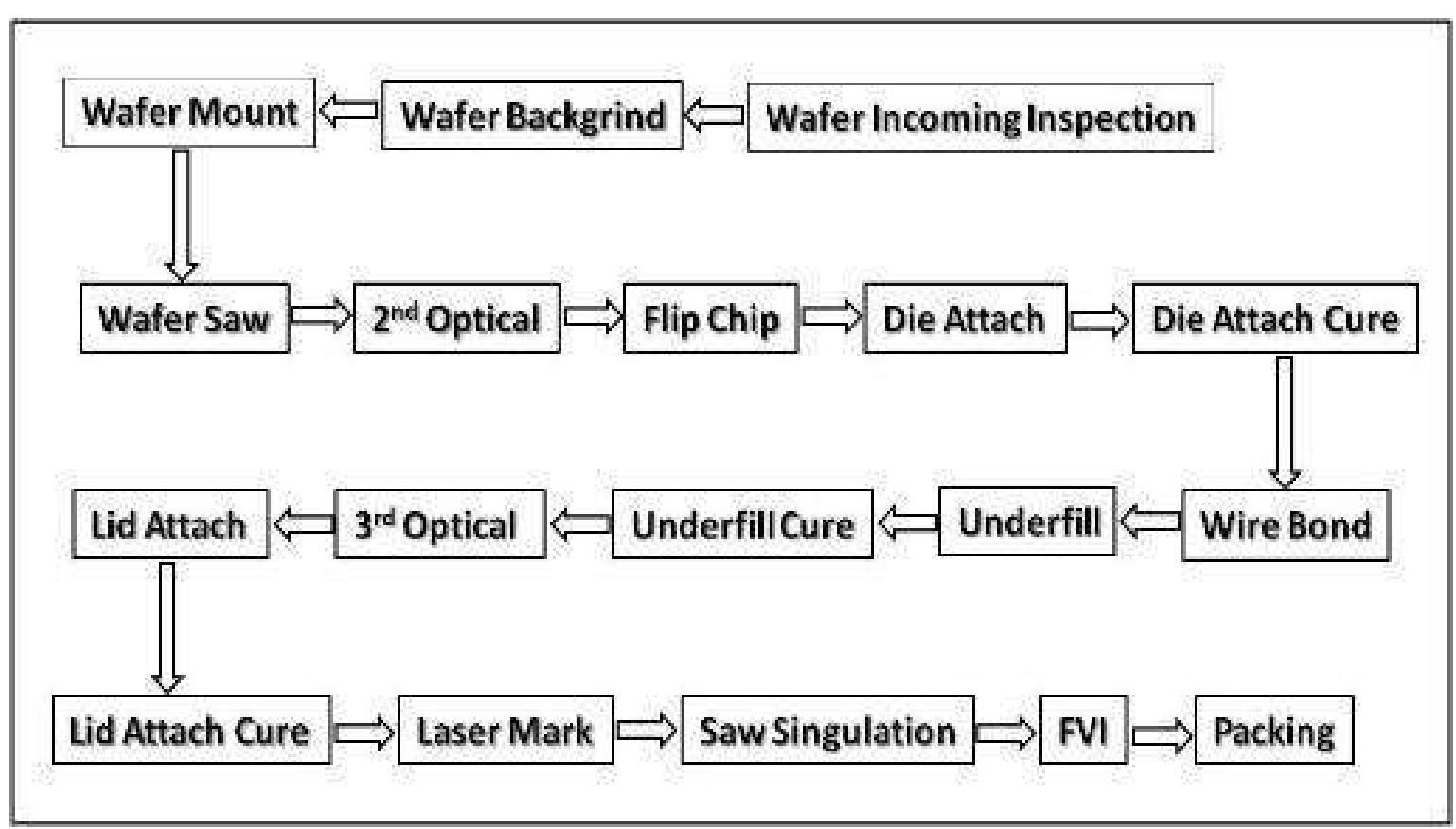

Figure 2. Flowchart for IC LGA Production. 
Table 3a. The Actual Capacity Per Every Single Process of LGA IC

\begin{tabular}{|c|c|c|c|c|c|c|c|c|c|}
\hline $\begin{array}{c}\text { No. } \\
\text { Sample }\end{array}$ & INCOMING & BACKGRIND & $\begin{array}{c}\text { WF } \\
\text { MOUNT }\end{array}$ & $\begin{array}{c}\text { WAFER } \\
\text { SAW }\end{array}$ & $\begin{array}{l}\text { 2ND } \\
\text { OPT }\end{array}$ & $\begin{array}{l}\text { Flip } \\
\text { Chip }\end{array}$ & $\begin{array}{c}\text { Die } \\
\text { Attach }\end{array}$ & $\begin{array}{c}\text { Die } \\
\text { Attach } \\
\text { Cure }\end{array}$ & $\begin{array}{l}\text { WIRE } \\
\text { BOND }\end{array}$ \\
\hline 1 & 10332 & 24145 & 21713 & 8145 & 10087 & 5092 & 3944 & 24394 & 1987 \\
\hline 2 & 10330 & 24238 & 22638 & 8090 & 10099 & 5059 & 3935 & 24381 & 1982 \\
\hline 3 & 10398 & 24288 & 21213 & 8141 & 10053 & 5101 & 3919 & 24396 & 1983 \\
\hline 4 & 10349 & 24161 & 21796 & 8123 & 10172 & 5071 & 3955 & 24393 & 1978 \\
\hline 5 & 10346 & 24149 & 21213 & 8141 & 100172 & 5071 & 3951 & 24386 & 1988 \\
\hline 6 & 10393 & 23950 & 22156 & 8100 & 10204 & 5047 & 3934 & 24389 & 1975 \\
\hline 7 & 10374 & 24126 & 22434 & 8118 & 10073 & 5046 & 3936 & 24388 & 1978 \\
\hline 8 & 10354 & 24365 & 21647 & 8121 & 10112 & 5021 & 3945 & 24391 & 1987 \\
\hline 9 & 10337 & 23928 & 22398 & 8117 & 10075 & 5081 & 3926 & 24380 & 1982 \\
\hline 10 & 10365 & 24376 & 22377 & 8078 & 10131 & 5057 & 3949 & 24379 & 1987 \\
\hline 11 & 10434 & 24512 & 21489 & 8131 & 10088 & 5072 & 3945 & 24379 & 1987 \\
\hline 12 & 10407 & 24042 & 22082 & 8088 & 10131 & 5029 & 3959 & 24394 & 1989 \\
\hline 13 & 10314 & 24042 & 22371 & 8093 & 10128 & 5038 & 3919 & 24395 & 1983 \\
\hline 14 & 10393 & 23936 & 22510 & 8086 & 10061 & 5089 & 3959 & 24386 & 1984 \\
\hline 15 & 10317 & 24164 & 22089 & 8155 & 10098 & 5038 & 3926 & 24378 & 1989 \\
\hline 16 & 10312 & 24407 & 22031 & 8110 & 10155 & 5082 & 3952 & 24379 & 1989 \\
\hline 17 & 10378 & 23986 & 21913 & 8095 & 10151 & 5080 & 3956 & 24390 & 1986 \\
\hline 18 & 10381 & 24022 & 21909 & 8131 & 10060 & 5080 & 3945 & 24380 & 1974 \\
\hline 19 & 10417 & 24229 & 21718 & 8101 & 10048 & 5061 & 3955 & 24387 & 1983 \\
\hline 20 & 10392 & 24185 & 21670 & 8088 & 10099 & 5060 & 3957 & 24391 & 1988 \\
\hline 21 & 10367 & 24490 & 21635 & 8126 & 10188 & 5083 & 3959 & 24389 & 1977 \\
\hline 22 & 10316 & 24313 & 22490 & 8140 & 10140 & 5050 & 3930 & 24379 & 1984 \\
\hline 23 & 10374 & 24010 & 22057 & 8106 & 10085 & 5019 & 3954 & 24388 & 1974 \\
\hline 24 & 10339 & 24305 & 21985 & 8128 & 10184 & 5039 & 3912 & 24395 & 1989 \\
\hline 25 & 10439 & 24117 & 22575 & 8137 & 10109 & 5118 & 3936 & 24380 & 1991 \\
\hline 26 & 10369 & 24526 & 21764 & 8125 & 10188 & 5054 & 3927 & 24385 & 1976 \\
\hline 27 & 10399 & 24196 & 22442 & 8108 & 10132 & 5084 & 3921 & 24389 & 1982 \\
\hline 28 & 10389 & 23753 & 21977 & 8116 & 10166 & 5099 & 3920 & 24387 & 1976 \\
\hline 29 & 10318 & 24190 & 21488 & 8091 & 10098 & 5078 & 3931 & 24387 & 1980 \\
\hline 30 & 10324 & 24263 & 21242 & 8126 & 10171 & 5088 & 3950 & 24396 & 1981 \\
\hline
\end{tabular}

Table 3b. The Actual Capacity Per Every Single Process of LGA IC

\begin{tabular}{ccccccccc}
$\begin{array}{c}\text { Under } \\
\text { Fill }\end{array}$ & $\begin{array}{c}\text { Under } \\
\text { Fill } \\
\text { Cure }\end{array}$ & $\begin{array}{c}\text { 3rd } \\
\text { OPT }\end{array}$ & $\begin{array}{c}\text { LID } \\
\text { ATTACH }\end{array}$ & $\begin{array}{c}\text { IA } \\
\text { Cure }\end{array}$ & MARKING & SAWSING & FVI & PACKING \\
\hline 3650 & 24520 & 4332 & 2564 & 25721 & 10399 & 3758 & 4350 & 6777 \\
3649 & 24521 & 4348 & 2564 & 25709 & 10343 & 3773 & 4270 & 6795 \\
3602 & 24521 & 4340 & 2559 & 25723 & 10420 & 3731 & 4224 & 6764 \\
3630 & 24529 & 4346 & 2577 & 25719 & 10374 & 3784 & 4374 & 6772 \\
3636 & 24512 & 4364 & 2573 & 25723 & 10418 & 3748 & 4359 & 6775 \\
3625 & 24517 & 4365 & 2563 & 25717 & 10392 & 3783 & 4273 & 6761 \\
3627 & 24524 & 4362 & 2570 & 25712 & 10383 & 3752 & 4338 & 6811 \\
3602 & 24526 & 4351 & 2573 & 25707 & 10375 & 3737 & 4331 & 6769 \\
3599 & 24521 & 4340 & 2582 & 25717 & 10330 & 3760 & 4297 & 6735 \\
3609 & 24521 & 4362 & 2557 & 25712 & 10420 & 3764 & 4345 & 6787 \\
3614 & 24521 & 4356 & 2560 & 25713 & 10350 & 3753 & 4341 & 6735 \\
3648 & 24526 & 4344 & 2564 & 25711 & 10369 & 3743 & 4304 & 6765 \\
3600 & 24518 & 4361 & 2580 & 25704 & 10405 & 3745 & 4334 & 6782 \\
3625 & 24529 & 4380 & 2579 & 25717 & 10371 & 3742 & 4258 & 6785 \\
3617 & 24513 & 4339 & 2567 & 25720 & 10399 & 3766 & 4356 & 6759 \\
3592 & 24525 & 4350 & 2583 & 25716 & 10359 & 3757 & 4291 & 6738 \\
3630 & 24530 & 4367 & 2572 & 25707 & 10408 & 3743 & 4302 & 6753 \\
3642 & 24527 & 4333 & 2571 & 25715 & 10353 & 3743 & 4236 & 6759 \\
\hline
\end{tabular}




\begin{tabular}{ccccccccc}
\hline \multicolumn{7}{c}{ Table 3b. The Actual Capacity Per Every Single Process of LGA IC } \\
\hline $\begin{array}{c}\text { Under } \\
\text { Fill }\end{array}$ & $\begin{array}{c}\text { Under } \\
\text { Fill } \\
\text { Cure }\end{array}$ & $\begin{array}{c}\text { 3rd } \\
\text { OPT }\end{array}$ & $\begin{array}{c}\text { LID } \\
\text { ATTACH }\end{array}$ & $\begin{array}{c}\text { IA } \\
\text { Cure }\end{array}$ & MARKING & SAWSING & FVI & PACKING \\
\hline 3642 & 24531 & 4355 & 2558 & 25716 & 10442 & 3766 & 4332 & 6773 \\
3628 & 24514 & 4352 & 2560 & 25713 & 10399 & 3776 & 4348 & 6794 \\
3609 & 24530 & 4366 & 2571 & 25714 & 10384 & 3765 & 4289 & 6763 \\
3609 & 24530 & 4366 & 2571 & 25714 & 10384 & 3765 & 4263 & 6815 \\
3636 & 24526 & 4358 & 2564 & 25710 & 10356 & 3768 & 4269 & 6752 \\
3621 & 24522 & 4371 & 2582 & 25713 & 10417 & 3740 & 4336 & 6746 \\
3648 & 24519 & 4343 & 2579 & 25707 & 10343 & 3759 & 4309 & 6768 \\
3620 & 24524 & 4338 & 2567 & 25716 & 10358 & 3747 & 4251 & 6731 \\
3623 & 24526 & 4376 & 2561 & 25712 & 10426 & 3756 & 4237 & 6788 \\
3639 & 24527 & 4361 & 2566 & 25712 & 10416 & 3757 & 4314 & 6781 \\
3635 & 24520 & 4377 & 2568 & 25710 & 10402 & 3742 & 4341 & 6804 \\
3629 & 24512 & 4346 & 2555 & 25720 & 10361 & 3733 & 4329 & 6800 \\
\hline
\end{tabular}

Table 4. Summary All The Unit Per Hour Machine

\begin{tabular}{cc}
\hline Station & UPH / Machine \\
\hline Incoming Inspection & 9329 \\
Backgrind & 21768 \\
Wafer Mount & 19811 \\
Ws & 7303 \\
2nd Opt & 9108 \\
Flip Chip & 4561 \\
Die Attach & 3546 \\
Die Attach Cure & 21948 \\
Wire Bond & 1785 \\
Under Fill & 3262 \\
Under Fill Cure & 22070 \\
3rd Opt & 3919 \\
Lid Attach & 2312 \\
LA Cure & 23142 \\
Marking & 9346 \\
Saw Sing & 3380 \\
Fvi & 3876 \\
Packing & 6094 \\
\hline
\end{tabular}

Below table $3 a-3 b$ is show the actual capacity per every single process of LGA IC. From above detail we can summary all the unit per hour machine capacity in below table 4.
Below is data of machine station, operator allocation and machine allocation for all process. 
Table 5. Data of Machine Station, Opertor Allocation and Machine Allocation for All Process

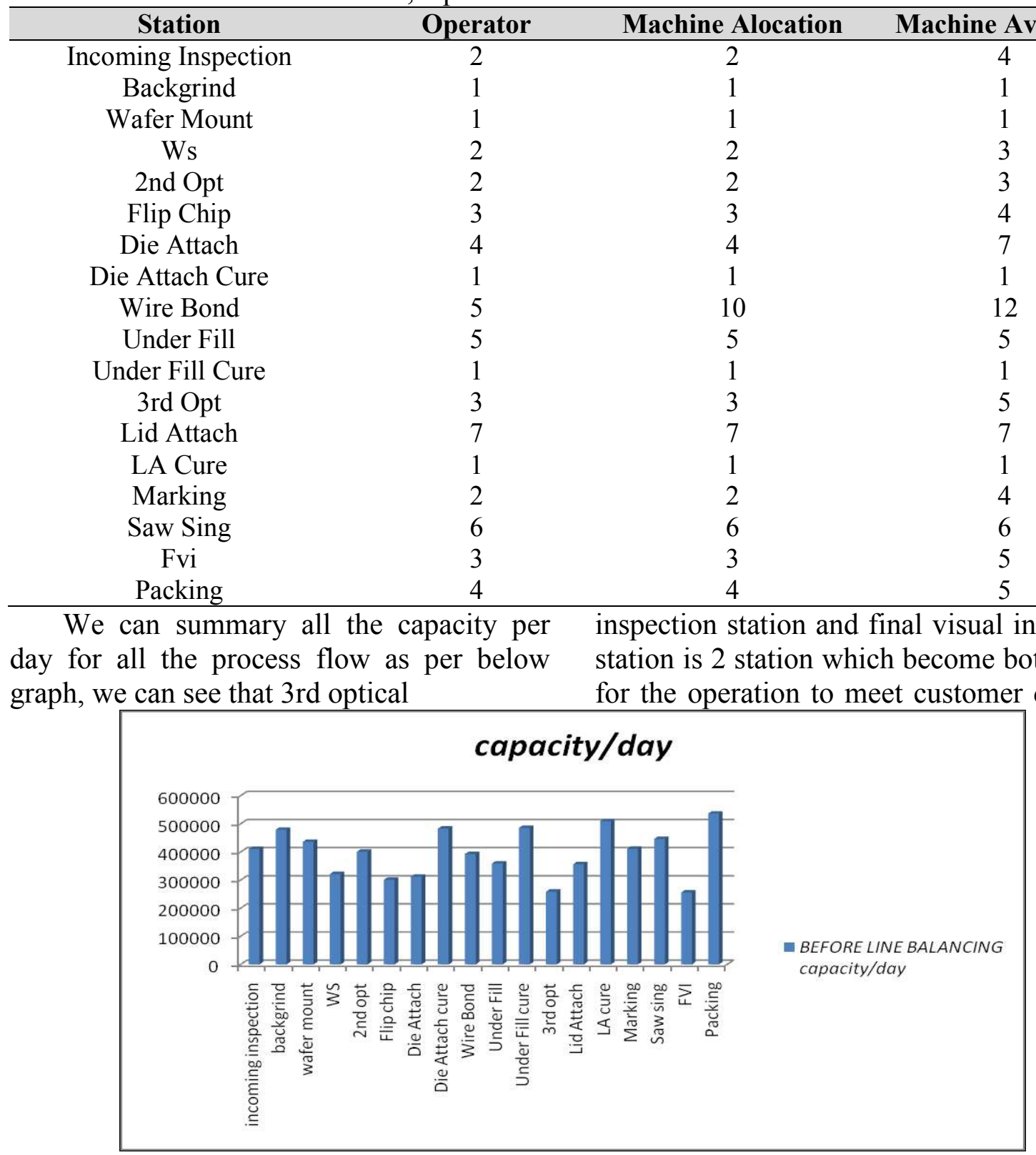

Figure 3. Capacity Per Day Before Line Balancing Chart.

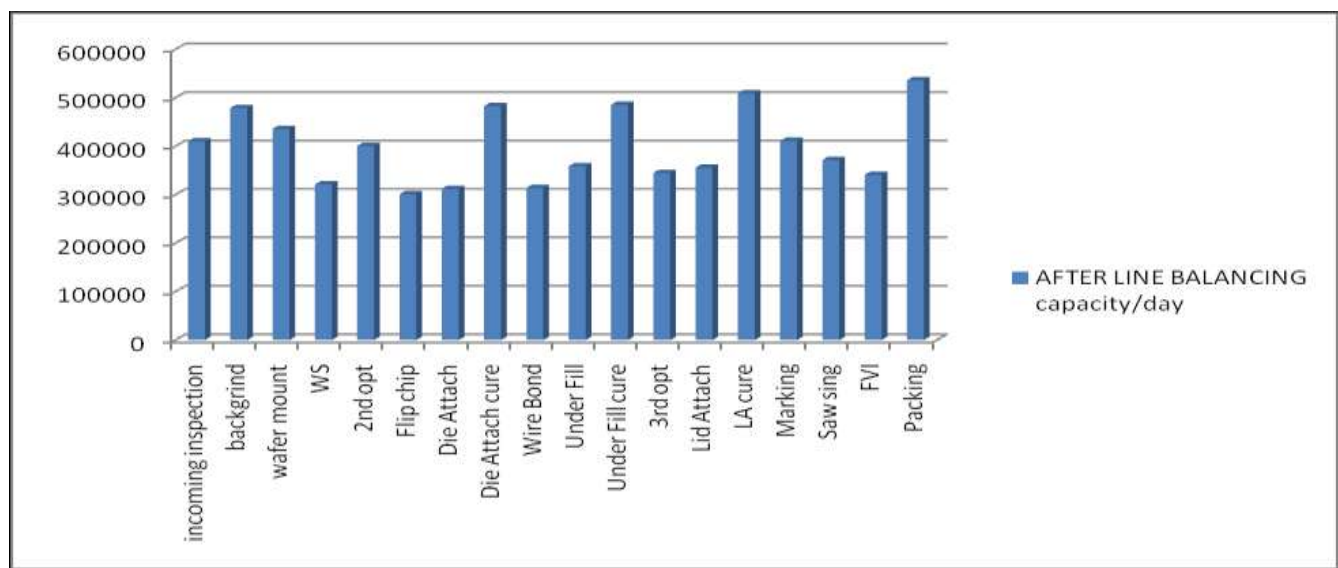

Figure 4. Capacity Per Day After Line Balancing Chart. 
After line balancing we can see below is capacity per day for all process flow on figure 3 and 4 . Base on existing condition, company not able to meet all customer demand which show in the following on time delivery data in below chart.

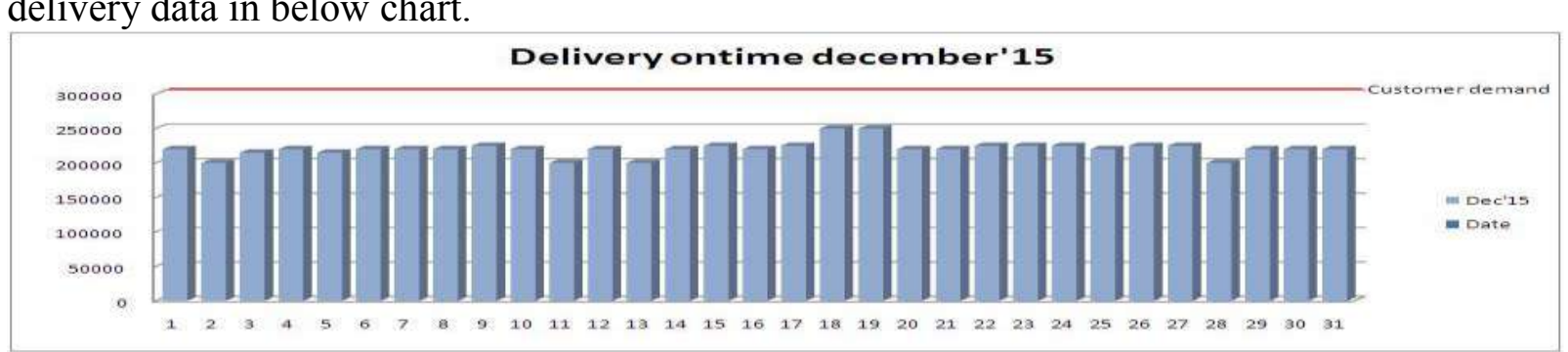

Figure 5. Delivery Ontime December 2015 Chart.

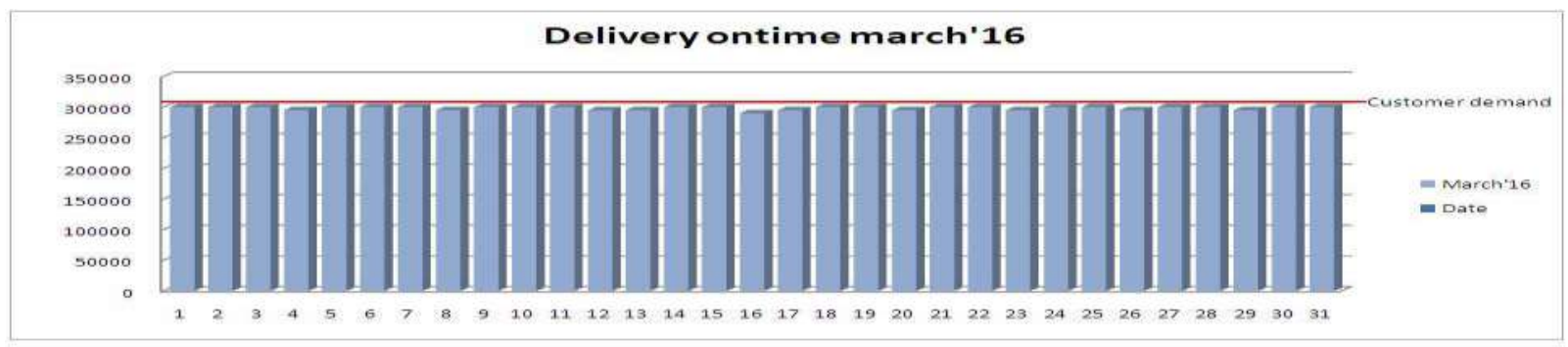

Figure 6. Delivery Ontime March 2015 Chart.

\section{CONCLUSION}

The research identify there are two station which impact alot of waiting the production process due to bottle neck capacity in 3rd optical inspection station and final visual inspection station. By applied line balancing methode as one of the Lean Manufacturing tools, we can increase the capacity in the two mension bottle neck. We can see the improvement by comparing on time delivery data before and after imrovement.

\section{REFERENCES}

A.M. Kilpatrick, Lean Manufacturing Principles : A comprehensive Framework for Improving Production Efficiency.
After the improvement, we can see that starting march 2016 company able to meet most of the customer demand which show in following chart. 